\title{
Kinetic temperatures in the Orion Bar
}

\author{
W. Batrla ${ }^{1}$ and T. L. Wilson ${ }^{2}$ \\ 1 Fachhochschule Hof, Abteilung Münchberg, Kulmbacher Strasse 76, 95213 Münchberg, Germany \\ 2 Max-Planck-Institut für Radioastronomie, Auf dem Hügel 69, 53121 Bonn, Germany \\ Received 9 April 2003 / Accepted 10 June 2003
}

\begin{abstract}
We report the detection of the $(J, K)=(1,1),(2,2)$ and $(4,4)$ inversion lines of ammonia towards the Photo Dissociation Region (PDR) of the Orion Bar. The $\mathrm{NH}_{3}$ emission is found in the same location as emission from HCN. Temperatures determined from line intensity ratios indicate a warm gas with $T_{\mathrm{k}}$ of $\sim 150 \mathrm{~K}$. This value is inconsistent with temperatures predicted from models of the gas phase chemistry of dense PDRs. We propose that the emission arises from ammonia released into the gas phase from the evaporation of dense clumps. From measurements of the $J=2-1$ line of $\mathrm{C}^{18} \mathrm{O}$, we obtain beam-averaged column densities for those positions where the $(1,1),(2,2)$ and $(4,4)$ inversion lines were measured. The ammonia to $\mathrm{C}^{18} \mathrm{O}$ abundance ratio is $1.4 \pm 0.5 \times 10^{-2}$.
\end{abstract}

Key words. ISM: clouds - ISM: individual objects: Orion - ISM: molecules - radio lines: ISM

\section{Introduction}

Photon Dominated Regions (PDRs) are those in which the UV radiation field is many times the average field found near the Sun. This radiation is provided by nearby $\mathrm{O}$ and $\mathrm{B}$ stars. Because of the intense UV fields, PDRs contain atoms in various ionization states, and only those molecules which are dissociated at higher energies. In our galaxy and other galaxies, PDRs give rise to prominent emission in spectral lines of $\mathrm{CO}$ in the millimeter and IR wavelength ranges and also in broadband dust emission. The heating of gas phase constituents in PDRs is thought to involve the photoejection of electrons from grains, with the transfer of energy to gas constituents. To reach the high temperatures found in PDRs, there must be a population of small grains. PDR models must include a complete description of the region involved. These include chemistry, interaction of dust and gas phases, and the thermal balance of the PDR. The models are characterized by the parameters $G_{0}$, the photon flux as a fraction of the photon field near the Sun, and $n$, the local density. Two additional factors, namely geometry and time dependence, must be taken into account. A number of details of PDR models are not yet fully understood. This requires detailed modelling and measurement of nearby regions. On the basis of refined models, one can determine parameters of PDRs in galaxies (see Hollenbach \& Tielens 1999; Kaufman et al. 1999).

One of the best-studied PDRs is the Orion Bar. This is an edge-on feature to the south west of the Trapezium (see Tauber et al. 1995; Hogerheijde et al. 1995; Young Owl et al. 2000). Due to the very favorable geometry; one can follow the distribution of atomic and molecular species as a function of depth

Send offprint requests to: W. Batrla, e-mail: wbatrla@fhs.fh-hof.de into the PDR. One of the parameters predicted by PDR models is the kinetic temperature, $T_{\mathrm{k}}$, as a function of depth into the region. Estimates of $T_{\mathrm{k}}$ for PDRs have been made using the peak temperatures of $\mathrm{CO}$ rotational transitions. However the peak temperatures of these lines are directly affected by beam dilution. A better method to estimate $T_{\mathrm{k}}$ would be to use ratios of lines so that beam dilution would have no effect. The ratios of $\mathrm{NH}_{3}$ inversion-rotation lines offer an alternative method to determine $T_{\mathrm{k}}$.

The rotation-inversion lines of the $\mathrm{NH}_{3}$ molecule are emitted at $\sim 1 \mathrm{~cm}$. With one receiver, we can measure a number of such lines emitted from levels at a wide range of temperatures above the ground state. Each of these lines is split into 5 groups of quadrupole hyperfine components which are usually emitted in Local Thermodynamic Equilibrium (LTE). From the ratios of these lines, we can determine the optical depth of the transition. The populations of metastable $(J=K)$ rotation-inversion lines are exchanged by collisions, so that the relative populations are characterized by a temperature, $T_{\text {rot }}$, which is closely related to $T_{\mathrm{k}}$. Since ratios are involved, $T_{\text {rot }}$ values do not depend on corrections for telescope beamsize, although the value of $T_{\text {rot }}$ is averaged over the beam. The value of $T_{\text {rot }} \leq T_{\mathrm{k}}$. This is because the collisions tend to preferentially transfer populations from higher to lower energy levels. A density-dependent correction is needed to relate $T_{\text {rot }}$ and $T_{\mathrm{k}}$. Reliable estimates of the correction are available (Danby et al. 1988).

In order to provide additional tests for PDR models, we have carried out measurements of $\mathrm{NH}_{3}$ inversion-rotation lines for the Orion Bar region using the Effelsberg 100-m telescope.

\section{Observations}

Observations were performed employing the single channel maser receiver and the 1024-channel autocorrelation 


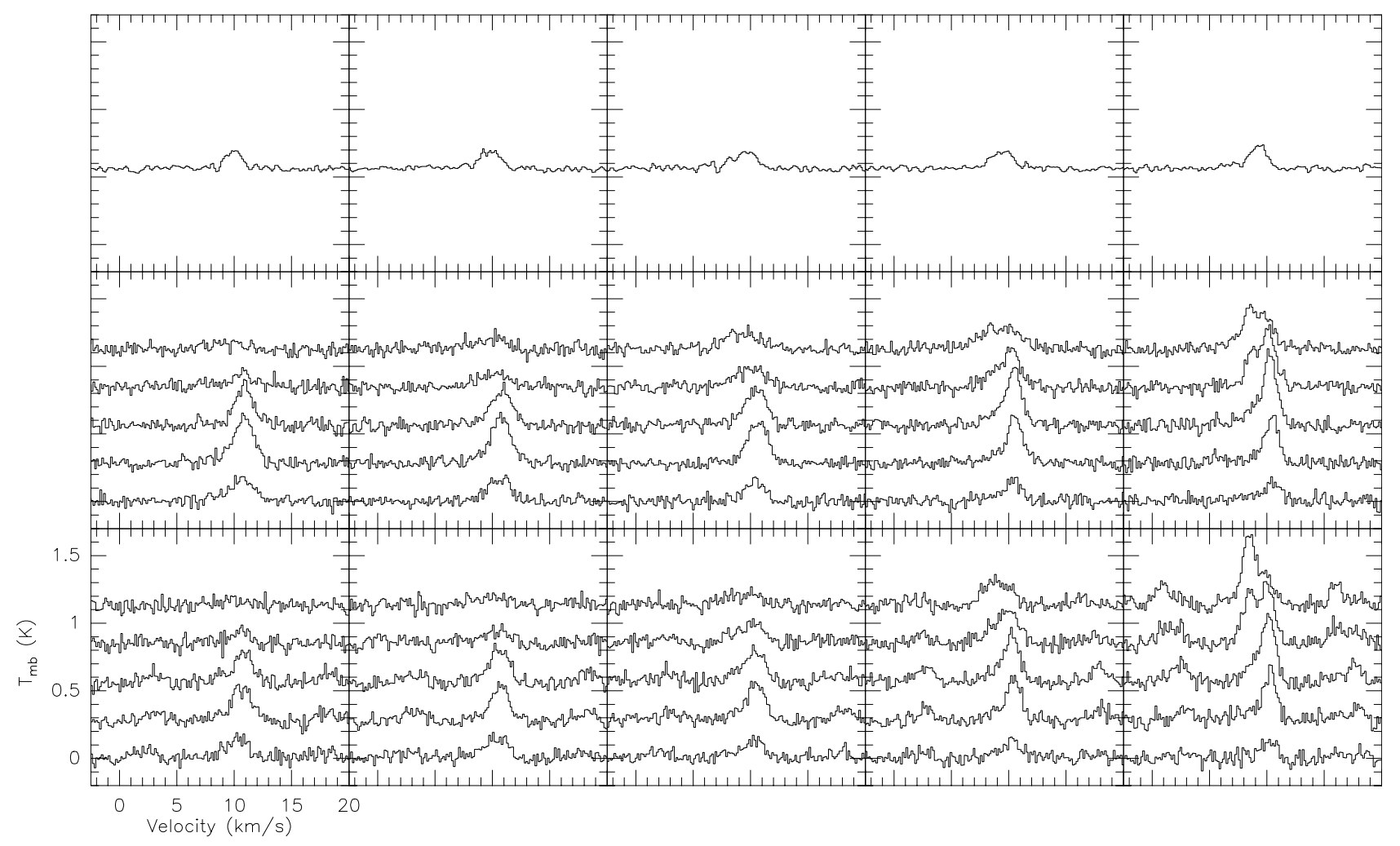

Fig. 1. The $(J, K)=(1,1)$, bottom, $(2,2)$, middle, and $(4,4)$, top row, inversion line spectra taken toward the Orion Bar. Panels are arranged from north east to south west in one row. The spectra along a cut across the bar from south east to north west are stacked in one panel.

spectrometer. Splitting the backend into two subunits of $6.25 \mathrm{MHz}$ each the $(1,1)$ and $(2,2)$ lines at the line rest frequencies of $23694.496 \mathrm{MHz}$ respectively $23722.631 \mathrm{MHz}$ were observed simultaneously, resulting in a velocity resolution of $0.151 \mathrm{~km} \mathrm{~s}^{-1}$ centered at a VLSR of $9.0 \mathrm{~km} \mathrm{~s}^{-1}$. We observed the $(4,4)$ line (rest frequency $24139.417 \mathrm{MHz}$ ) with the 1024-channel Autocorrelator used as two 512 channel spectrometers, and the line centered at VLSR $9.0 \mathrm{~km} \mathrm{~s}^{-1}$ in both subunits. Here, spectra in both halves from the backend were added prior to data reduction.

System temperature under clear sky at zenith was of the order of $50 \mathrm{~K}$ over a bandwidth of $80 \mathrm{MHz}$, while at the low elevation of Orion at Effelsberg and depending on weather the system temperature was typically in the range of 80 to $120 \mathrm{~K}$. We tied the temperature scale to the primary calibrator NGC 7027 using a flux density $6.0 \mathrm{mJy}$ at the wavelength of $1.3 \mathrm{~cm}$. The HPBW of the telescope at this wavelength is $43^{\prime \prime}$. Pointing constants were determined at the beginning of an observing period and every two to three hours thereafter. We found the pointing to be accurate to 5 " rms.

We employed position switching with an off-source integration followed by an on-source integration of $3 \mathrm{~min}$ duration each, with the reference position 3 min of time west of the source position. Total integration time ranged between 18 and 30 min per position.

Spectra of the $(1,1)$ and $(2,2)$ lines were observed on a rectangular 5 point by 5 point raster, which was rotated by 45 degrees West of North. The spacing between the raster points was $20^{\prime \prime}$. The $(4,4)$ line was observed only toward the central line of this raster parallel to the ionization front. The positions observed are marked by crosses in Fig. 2. In Tables 1 and 2 the offset rounded to full seconds of arc for the observed positions are given relative to the reference position of Hogerheijde et al. (1995), namely $\alpha=05^{\mathrm{h}} 32^{\mathrm{m}} 52.7^{\mathrm{s}}, \delta=-05^{\circ} 27^{\prime} 00^{\prime \prime}(1950.0)$.

We carried out additional measurements of the $J=2-1$ line of $\mathrm{C}^{18} \mathrm{O}$ for those positions where spectra of the $(1,1)$, $(2,2)$ and $(4,4)$ inversion lines were taken. These data were taken with the 10-meter Heinrich-Hertz-Telescope (HHT) on Mt. Graham AZ. The telescope beam size was $35^{\prime \prime}$ at the line frequency. The SIS mixer is a double sideband system, so the line intensities were calibrated using the peak $\mathrm{C}^{18} \mathrm{O}$ temperature measured for Orion KL (see e.g., Wilson et al. 1999 for a fuller discussion of this procedure). The SIS receiver noise temperature was $\sim 380 \mathrm{~K}$, the spectrometers were Acoustic Optical Spectrometers and filter banks.

\section{Results}

We show all the spectra of the three $\mathrm{NH}_{3}(J, K)=(1,1),(2,2)$, and $(4,4)$ inversion lines, in Fig. 1 . The distribution of the $\mathrm{NH}_{3}$ shows a narrow ridge parallel but offset to the south east from the ionization front outlined in Fig. 2. This line is centered at a radial velocity of about $10.5 \mathrm{~km} \mathrm{~s}^{-1}$. In the south west of the raster a second line is visible in the two spectra taken north west of the ionization front. This feature is at a radial velocity of $8.5 \mathrm{~km} \mathrm{~s}^{-1}$, which is characteristic for ammonia in the Orion ridge (see Cesaroni \& Wilson 1994), and, judging from the relative strength of the $(1,1)$ and $(2,2)$ lines, seems to arise from gas of a different temperature. This assumption is supported by 
Table 1. Measured $\mathrm{NH}_{3}$ line parameters.

\begin{tabular}{|c|c|c|c|c|c|c|c|c|c|}
\hline $\begin{array}{r}(1) \\
\text { offset }\end{array}$ & $\begin{array}{r}(2) \\
T_{\mathrm{MB}} \\
(\mathrm{K})\end{array}$ & $\begin{array}{r}(3) \\
\Delta V_{1 / 2} \\
\left(\mathrm{~km} \mathrm{~s}^{-1}\right)\end{array}$ & $\begin{array}{r}(4) \\
V_{\mathrm{LSR}} \\
\left(\mathrm{km} \mathrm{s}^{-1}\right)\end{array}$ & $\begin{array}{r}(5) \\
T_{\mathrm{MB}} \\
(\mathrm{K})\end{array}$ & $\begin{array}{r}(6) \\
\Delta V_{1 / 2} \\
\left(\mathrm{~km} \mathrm{~s}^{-1}\right)\end{array}$ & $\begin{array}{r}(7) \\
V_{\mathrm{LSR}} \\
\left(\mathrm{km} \mathrm{s}^{-1}\right)\end{array}$ & $\begin{array}{r}(8) \\
T_{\mathrm{MB}} \\
(\mathrm{K})\end{array}$ & $\begin{array}{r}(9) \\
\Delta V_{1 / 2} \\
\left(\mathrm{~km} \mathrm{~s}^{-1}\right)\end{array}$ & $\begin{array}{r}(10) \\
V_{\mathrm{LSR}} \\
\left(\mathrm{km} \mathrm{s}^{-1}\right)\end{array}$ \\
\hline $49,-19$ & 0.15 & $2.2 \pm 0.4$ & $10.3 \pm 0.1$ & 0.22 & $2.6 \pm 0.2$ & $10.6 \pm 0.1$ & - & - \pm- & - \pm- \\
\hline $34,-5$ & 0.15 & $2.5 \pm 0.3$ & $10.3 \pm 0.1$ & 0.17 & $2.4 \pm 0.2$ & $10.6 \pm 0.1$ & - & - \pm- & - \pm- \\
\hline 19,10 & 0.13 & $2.4 \pm 0.5$ & $10.2 \pm 0.2$ & 0.15 & $2.0 \pm 0.2$ & $10.4 \pm 0.1$ & 0.14 & $1.6 \pm 0.1$ & $10.9 \pm 0.1$ \\
\hline 5,24 & 0.12 & $2.3 \pm 0.5$ & $10.2 \pm 0.2$ & 0.15 & $1.9 \pm 0.2$ & $10.3 \pm 0.1$ & - & - \pm- & - \pm- \\
\hline$-9,38$ & 0.13 & $1.6 \pm 0.3$ & $10.2 \pm 0.1$ & 0.13 & $2.2 \pm 0.3$ & $10.4 \pm 0.1$ & - & - \pm- & - \pm- \\
\hline $34,-33$ & 0.24 & $2.1 \pm 0.3$ & $10.6 \pm 0.1$ & 0.33 & $2.1 \pm 0.1$ & $10.7 \pm 0.1$ & - & $\overline{- \pm-}$ & - \pm- \\
\hline $19,-19$ & 0.26 & $1.8 \pm 0.2$ & $10.7 \pm 0.1$ & 0.34 & $2.2 \pm 0.1$ & $10.6 \pm 0.1$ & - & - \pm- & - \pm- \\
\hline $5,-5$ & 0.27 & $2.0 \pm 0.2$ & $10.4 \pm 0.1$ & 0.31 & $2.1 \pm 0.1$ & $10.6 \pm 0.1$ & 0.13 & $2.1 \pm 0.1$ & $9.8 \pm 0.1$ \\
\hline$-9,10$ & 0.31 & $1.6 \pm 0.2$ & $10.4 \pm 0.1$ & 0.33 & $1.8 \pm 0.1$ & $10.5 \pm 0.1$ & - & - \pm- & - \pm- \\
\hline$-24,24$ & 0.37 & $1.4 \pm 0.2$ & $10.2 \pm 0.1$ & 0.33 & $1.5 \pm 0.1$ & $10.4 \pm 0.1$ & - & - \pm- & - \pm- \\
\hline $19,-47$ & 0.22 & $2.0 \pm 0.3$ & $10.8 \pm 0.1$ & 0.29 & $1.9 \pm 0.1$ & $10.9 \pm 0.1$ & - & $\overline{- \pm-}$ & - \pm- \\
\hline $5,-33$ & 0.25 & $2.0 \pm 0.2$ & $10.6 \pm 0.1$ & 0.25 & $2.3 \pm 0.1$ & $10.8 \pm 0.1$ & - & - \pm- & - \pm- \\
\hline$-9,-19$ & 0.22 & $2.4 \pm 0.3$ & $10.2 \pm 0.1$ & 0.27 & $2.2 \pm 0.1$ & $10.4 \pm 0.1$ & 0.12 & $2.4 \pm 0.1$ & $9.5 \pm 0.1$ \\
\hline$-24,-5$ & 0.33 & $1.8 \pm 0.3$ & $10.2 \pm 0.1$ & 0.39 & $1.9 \pm 0.1$ & $10.4 \pm 0.1$ & - & - \pm- & - \pm- \\
\hline$-38,10$ & 0.46 & $1.8 \pm 0.2$ & $10.0 \pm 0.1$ & 0.49 & $1.7 \pm 0.1$ & $10.2 \pm 0.1$ & - & - \pm- & - \pm- \\
\hline $5,-61$ & 0.11 & $1.7 \pm 0.4$ & $10.5 \pm 0.2$ & 0.12 & $2.4 \pm 0.1$ & $9.5 \pm 0.1$ & - & - \pm- & - \pm- \\
\hline$-9,-47$ & 0.09 & $3.1 \pm 0.6$ & $10.3 \pm 0.3$ & 0.10 & $3.3 \pm 0.3$ & $10.1 \pm 0.2$ & - & - \pm- & - \pm- \\
\hline$-24,-33$ & 0.14 & $3.2 \pm 0.4$ & $9.9 \pm 0.2$ & 0.14 & $3.2 \pm 0.2$ & $9.9 \pm 0.1$ & 0.13 & $2.3 \pm 0.1$ & $9.4 \pm 0.1$ \\
\hline$-38,-19$ & 0.21 & $2.1 \pm 0.3$ & $10.2 \pm 0.3$ & 0.22 & $1.5 \pm 0.2$ & $10.4 \pm 0.1$ & - & - \pm- & - \pm- \\
\hline$-52,-5$ & 0.37 & $1.4 \pm 0.3$ & $10.1 \pm 0.2$ & 0.43 & $1.5 \pm 0.1$ & $10.2 \pm 0.1$ & - & - \pm- & - \pm- \\
\hline$-9,-75$ & 0.04 & $4.7 \pm 1.8$ & $9.8 \pm 0.6$ & 0.05 & $3.6 \pm 0.6$ & $9.2 \pm 0.3$ & - & - \pm- & - \pm- \\
\hline$-24,-61$ & 0.07 & $4.3 \pm 0.8$ & $9.8 \pm 0.3$ & 0.08 & $3.4 \pm 0.4$ & $9.7 \pm 0.2$ & - & - \pm- & - \pm- \\
\hline$-38,-47$ & 0.09 & $4.3 \pm 0.7$ & $9.3 \pm 0.3$ & 0.12 & $3.7 \pm 0.3$ & $9.3 \pm 0.1$ & 0.17 & $1.8 \pm 0.1$ & $9.2 \pm 0.1$ \\
\hline$-52,-33$ & 0.10 & $1.5 \pm 0.8$ & $10.2 \pm 0.6$ & 0.15 & $4.2 \pm 0.3$ & $9.0 \pm 0.2$ & - & - \pm- & - \pm- \\
\hline$-66,-19$ & 0.21 & $1.6 \pm 1.1$ & $10.0 \pm 0.5$ & 0.13 & $1.3 \pm 0.3$ & $10.3 \pm 0.2$ & - & - \pm- & - \pm- \\
\hline
\end{tabular}

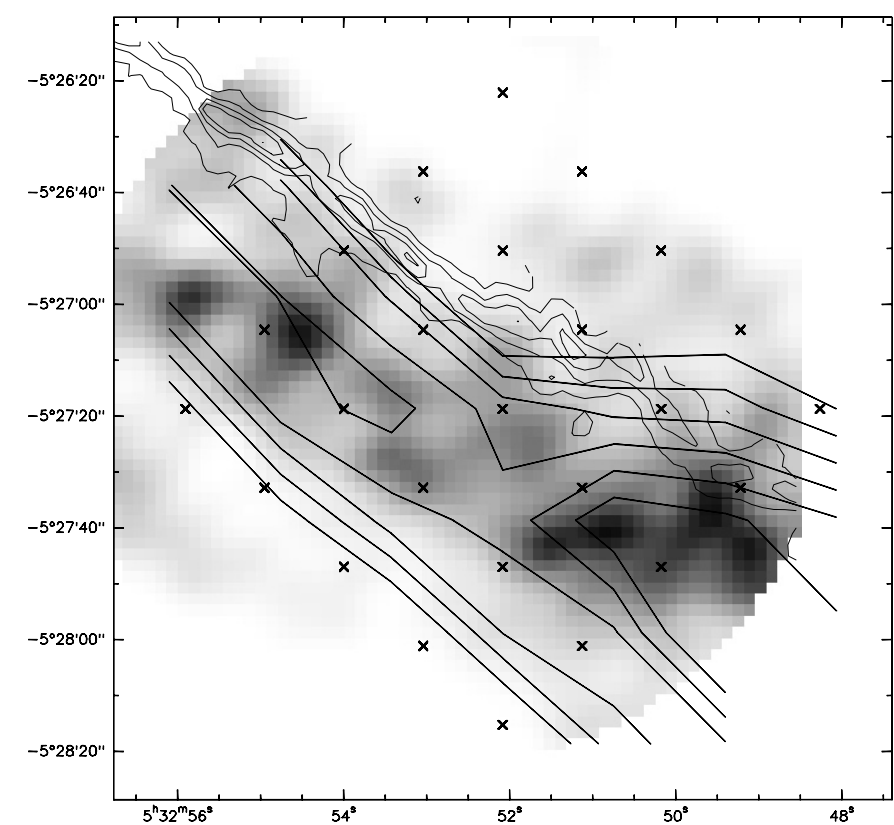

Fig. 2. Thick contours show the integrated intensity of the $(J, K)=(2,2)$ inversion line of ammonia in the interval from 9.5 to $11.5 \mathrm{~km} \mathrm{~s}^{-1}$. Crosses mark the positions observed. Contours start at $0.35 \mathrm{~K} \mathrm{~km} \mathrm{~s}^{-1}$ with contour intervals of $0.05 \mathrm{~K} \mathrm{~km} \mathrm{~s}^{-1}$. The gray scale image shows the integrated line intensities of the HCN emission from the Bar (Young Owl et al. 2000, their Fig. 5), taken with 9" resolution. Thin contours show the OI line intensity measured by Walmsley et al. (2000) to outline the location of the ionization front. Coordinates are RA, Dec 1950.0.

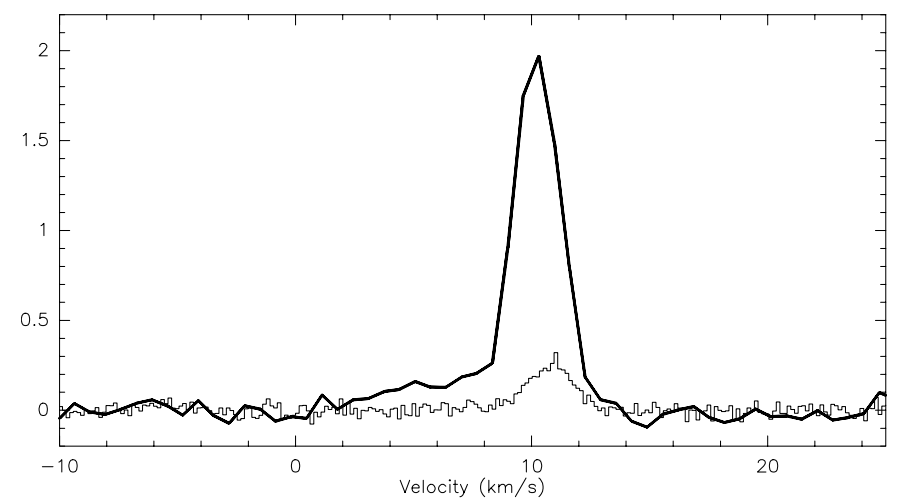

Fig. 3. The heavy line shows the spectrum of the $J=2-1$ line of $\mathrm{C}^{18} \mathrm{O}$ taken at the $(5,-5)$ offset position. This line allows one to determine the total column density of $\mathrm{C}^{18} \mathrm{O}$ for a large range of $T_{\mathrm{k}}$ and $n\left(\mathrm{H}_{2}\right)$. Thin line shows for comparison the $\mathrm{NH}_{3}(2,2)$ line at the same position. Temperature scale is identical for both spectra.

the absence of the latter feature in the $(4,4)$ line. Therefore, in this paper, we will concentrate on the line at $10.5 \mathrm{~km} \mathrm{~s}^{-1}$ solely.

The absence of the hyperfine components in all lines indicates that optical depths are low. Therefore, only Gaussian fit results for all lines are given in Table 1 . Radial velocities and linewidths from our fits to the $(1,1)$ inversion lines show good agreement with corresponding parameters from our fits to the $(2,2)$ and $(4,4)$ inversion lines at the identical positions. 
Table 2. $T_{\text {rot }}$ from $\mathrm{NH}_{3}$ line ratios.

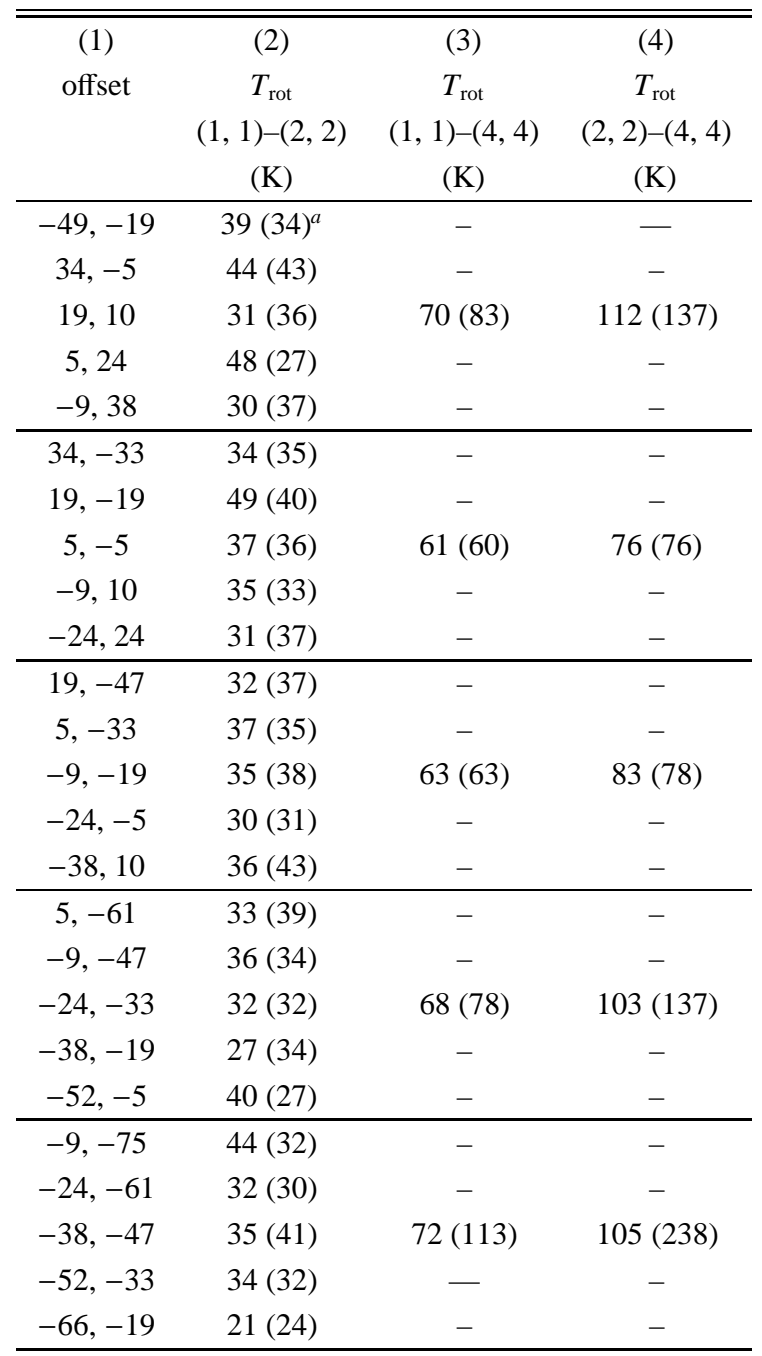

a Temperatures in parenthesis were calculated from $T_{\mathrm{mb}}$ under the assumption of equal linewidth.

From fitting a Gaussian profile to the amplitudes of one row of 5 spectra cutting perpendicular across the ionization front (the spectra contained in one panel of Fig. 1) we find that the maximum intensity is displaced between $23^{\prime \prime}$ and $25^{\prime \prime}$ south east of the ionization front.

The ratios of $\mathrm{NH}_{3}$ column densities can be used to obtain the rotational temperature, $T_{\text {rot }}$. We have calculated $T_{\text {rot }}$ values in Table 2. $T_{\text {rot }}$ is a lower limit to the actual kinetic temperature, $T_{\mathrm{k}}$. In particular, $T_{\text {rot }}$ values from the $(1,1)$ and $(2,2)$ inversion lines are close to the upper limit which one can estimate from these data alone. Where we have $(4,4)$ inversion line data, we can calculate the value of $T_{\text {rot }}$ from $(2,2)$ and $(4,4)$ inversion lines. As expected, for these, the $T_{\text {rot }}$ values are much larger; this shows that for such warm gas one needs data from higher lying energy levels, such as the $(4,4)$. The $T_{\text {rot }}$ values from the $(2,2)$ and $(4,4)$ inversion lines are more accurate and are to be preferred. $T_{\text {rot }}$ is proportional to $T_{\mathrm{k}}$, but the relation depends on the $\mathrm{H}_{2}$ density and the abundance of $\mathrm{NH}_{3}$. If we use a typical relation from Danby et al. (1988), for a density of $2 \times 10^{4} \mathrm{~cm}^{-3}$,
Table 3. $T_{\text {rot }}$, total $\mathrm{NH}_{3}$ column densities and $\mathrm{NH}_{3} / \mathrm{C}^{18} \mathrm{O}$ ratios.

\begin{tabular}{cccc}
\hline \hline $\begin{array}{c}(1) \\
\text { offset }\end{array}$ & $\begin{array}{c}(2) \\
T_{\text {rot }} \\
\mathrm{K}\end{array}$ & $\begin{array}{c}(3) \\
N\left(\mathrm{NH}_{3}\right) \\
\mathrm{cm}^{-2}\end{array}$ & $\begin{array}{c}(4) \\
\left(\mathrm{NH}_{3}\right) / N\left(\mathrm{C}^{18} \mathrm{O}\right)\end{array}$ \\
\hline 19,10 & 112 & $4.3(13)$ & $1.6(-2)$ \\
$5,-5$ & 76 & $6.0(13)$ & $1.8(-2)$ \\
$-9,-19$ & 83 & $6.4(13)$ & $1.9(-2)$ \\
$-24,-33$ & 103 & $6.1(13)$ & $1.0(-2)$ \\
$-38,-47$ & 105 & $5.2(13)$ & $9.0(-3)$ \\
\hline
\end{tabular}

a $T_{\text {rot }}$ of $90 \mathrm{~K}$ to $110 \mathrm{~K}$ yields a $T_{\mathrm{k}}$ value of $150 \mathrm{~K}$ to $220 \mathrm{~K}$. The conversion becomes smaller for higher densities, but that given by Danby et al. (1988) will provide a reasonably correct value of $T_{\mathrm{k}}$.

Using the $\mathrm{NH}_{3}$ for the 5 positions where we have measured $(4,4)$ inversion lines, we have used Eq. (14.66) of Rohlfs \& Wilson (1999) to calculate the total column densities of $\mathrm{NH}_{3}$. These are given in Col. 3 of Table 3 . We have carried out measurements of the $J=2-1$ line of $\mathrm{C}^{18} \mathrm{O}$ at these positions with the HHT. From an LVG analysis, one can obtain a reliable estimate of the total column density of $\mathrm{C}^{18} \mathrm{O}$ from the $J=2-1$ line for $T_{\mathrm{k}}$ between 15 and $80 \mathrm{~K}$, and $\mathrm{H}_{2}$ densities between $10^{3}$ and $10^{6} \mathrm{~cm}^{-3}$. From a modification of Eq. (14.115) of Rohlfs $\&$ Wilson (1999), the column density of $\mathrm{C}^{18} \mathrm{O}$ is:

$N\left(\mathrm{C}^{18} \mathrm{O}\right)=5.3 \times 10^{14} \int T_{\mathrm{MB}}\left(\mathrm{C}^{18} \mathrm{O}, J=2-1\right) \mathrm{d} v$

It is possible that the $\mathrm{C}^{18} \mathrm{O}$ and $\mathrm{NH}_{3}$ are not completely coextensive. However, the linewidths and radial velocities are in good agreement. A more detailed comparison would require very high resolution maps of both species. For the present, we must assume that the $\mathrm{NH}_{3}$ and $\mathrm{C}^{18} \mathrm{O}$ arise in the same region. Then one can determine relative abundances. These are given in Col. 4 of Table 3 . The $\mathrm{NH}_{3} / \mathrm{C}^{18} \mathrm{O}$ ratios range from $1.9 \times 10^{-2}$ to $9.0 \times 10^{-3}$, with an average value of $1.4 \pm 0.5 \times 10^{-2}$. This is a value somewhat lower than found in molecular clouds, but is probably an indication of the high UV fields in this environment.

\section{Discussion}

The region where we measured our $\mathrm{NH}_{3}$ data has been studied in a large number of molecular transitions by Hogerheijde et al. (1995), who took spectra at a raster line running from north west to south east perpendicular across the ionization front. Comparisons with their data are straightforward. Our position at offset $(5,-5)$ is just 7 " to the south east of their reference position. So the spectra in the panels of column two in Fig. 1 can be directly compared with the spectra in Fig. 2 of Hogerheijde et al. (1995), especially the three lines of HCN. Both Nitrogen bearing molecules show a maximum at identical positions. We make an additional comparison in Fig. 2, where we superpose the line integral of the $\mathrm{NH}_{3}(2,2)$ line, shown as thick contours, on the $\sim 9^{\prime \prime}$ resolution HCN $J=1-0$ integrated line map, shown as grey scale (Young Owl et al. 2000). 
Thin contours show the location of the ionization front as outlined by the OI-line (Walmsley et al. 2000).

The agreement between the spatial distributions of $\mathrm{HCN}$ and $\mathrm{NH}_{3}$ is very good, so we conclude that our $\mathrm{NH}_{3}$ emission arises from the Orion Bar region, and there appears to be little or no offset from the HCN maxima of Young Owl et al. (2000). Thus the $\mathrm{NH}_{3}$ emission originates from that part of the Orion Bar that contains HCN. The models of both Hogerheijde et al. (1955) and Young Owl et al. (2000) for the Orion Bar PDR require two components, clumps with a $\mathrm{H}_{2}$ density $\sim 10^{6}$ and an interclump medium of density $\sim 5 \times 10^{4}$. The high resolution data indicate that most of the $\mathrm{HCN}$ emission arises deep within the clumps. We assume that the $\mathrm{NH}_{3}$ also arises from the high density clumps, and consider that our detection of $\mathrm{NH}_{3}$ is an indirect argument for clumping. Sternberg \& Dalgarno (1995) model the gas phase chemistry in a dense (albeit plane parallel) PDR. According to their model $\mathrm{NH}_{3}$ does not exist in the interclump medium. The $\mathrm{NH}_{3}$ column densities and abundances derived above are consistent with the model if the emission arises at an $A_{\mathrm{v}}$ of about $8 \mathrm{mag}$. Temperatures predicted at that depth into the PDR are of the order of $20 \mathrm{~K}$. This is inconsistent with the temperatures determined from $\mathrm{NH}_{3}$. Another discrepancy for the $J=6-5$ line of $\mathrm{C}^{18} \mathrm{O}$ from the Orion Bar was found by Lis et al. (1997). Both of these results indicate that the PDR models need further refinement.

Gorti \& Hollenbach (2002) have modelled the twophase PDR medium in the Orion Bar and find that the best fit to the molecular data would have a kinetic temperature of $\sim 50 \mathrm{~K}$, deep inside the clumps, consistent with the value of $20 \mathrm{~K}$ but much less than the surface temperature of thousands of K. The model predicts photoevaporation of clumps (Gorti \& Hollenbach 2002), where more than $30 \%$ of the clump mass is lost. Hydrogenated species like $\mathrm{NH}_{3}$ are formed most efficiently by grain surface processes (see van Dieshoeck \& Blake 1998 and references therein). Release into the gas phase by evaporation of dense clumps seems a reasonable way for the production of the warm ammonia detected. The most likely location for this warm material would be the surfaces of the clumps of dense material toward the Trapezium cluster.

Since submitting this paper, an ODIN satellite measurement of the $J=1-0$ sub-mm rotational line of ortho- $\mathrm{NH}_{3}$ in the $K=0$ ladder at $572.5 \mathrm{GHz}$ has been published by Larsson et al. (A\&A 402, L69). Our data refer to para- $\mathrm{NH}_{3}$. However, with the assumption of Local Thermodynamic Equilibrium (LTE), we can use our results to determine the optical depth of the sub-mm $\mathrm{NH}_{3}$ line. Averaging over the 5 positions in Table 3, we find that the column density in the $(0,0)$ level is $4.9 \times 10^{13} \mathrm{~cm}^{-2}$. From Eqs. (14.38), (14.42) and (14.56) in Tools of Radio Astronomy, we find an optical depth of $\sim 1$ in the sub-mm line of $\mathrm{NH}_{3}$. The peak temperature of the $K=0, J=1-0$ line is $0.35 \mathrm{~K}, T_{\mathrm{MB}}$ in the ODIN $2^{\prime}$ beam. If we use our data in a plane parallel model for the Bar, we find that the geometric mean size of the $\mathrm{NH}_{3}$ emission region is $8^{\prime \prime}$. If the $\mathrm{NH}_{3}$ bar emission is 1.5 in size parallel to the Bar axis, the width of the emission region is $\sim 0$ ! $^{\prime} 8$.

\section{Conclusions}

We have identified weak $\mathrm{NH}_{3}$ inversion-rotation emission from the Orion Bar, a well-known PDR. Our measurements of $\mathrm{NH}_{3}$ rotation-inversion spectra have allowed a determination of the $T_{\text {rot }}$. We find an range of values of $60 \mathrm{~K}$ to $100 \mathrm{~K}$; that is, warm gas. The conversion of $T_{\text {rot }}$ to $T_{\mathrm{k}}$ depends on the local $\mathrm{H}_{2}$ density. For a typical model with density $2 \times 10^{4} \mathrm{~cm}^{-3}$, this would raise $T_{\text {rot }}=80 \mathrm{~K}$ to $T_{\mathrm{k}}=120 \mathrm{~K}$. A density value, however, depends on models of the PDR. The spatial distribution of the $\mathrm{NH}_{3}$ is very consistent with the location of the $\mathrm{HCN}$ emission. This is thought to arise from a series of clumps of density $>10^{6} \mathrm{~cm}^{-3}$, embedded in a $2 \times 10^{4} \mathrm{~cm}^{-3}$ interclump region. We identify the location of the $\mathrm{NH}_{3}$ emission as the surface layer in the clumps facing the Trapezium.

Acknowledgements. The authors thank C. M. Walmsley for providing a machine-readable copy of the map of the OI line towards the Orion Bar, as well M. Meixner for the map of the HCN line. Comments by the referee, D. Lis, helped to improve the presentation.

\section{References}

Cesaroni, R., \& Wilson, T. L. 1994, A\&A, 281, 209

Danby, G., Flower, D. R., Valiron, P., Schilke, P., \& Walmsley, C. M. 1988, MNRAS, 235, 229

Gorti, U., \& Hollenbach, D. J. 2002, ApJ, 573, 215

Hollenbach, D. J., \& Tielens, A. G. G. M. 1999, Rev. Mod. Phys., 71, 173

Hogerheijde, M. R., Jansen, D. J., \& van Dishoeck, E. F. 1995, A\&A, 294, 792

Kaufman, M. J., Wolfire, M., Hollenbach, D. J., \& Luhman, M. J. 1999, ApJ, 527, 795

Lis, D. C., Schilke, P., \& Keene, J. 1997, in CO: Twenty-Five Years of Millimeter Wave Spectroscopy, ed. W. B. Latter, S. J. E. Radford, P. R. Jewell, J. G. Mangum, \& J. Bally (Dordrecht: Kluwer), 128

Rohlfs, K., \& Wilson, T. L. 1999, Tools of Radio Astronomy (Heidelberg: Springer-Verlag)

Sternberg, A., \& Dalgarno, A. 1995, ApJS, 99, 565

Tauber, J. A., Lis, D. C., Keene, J., Schilke, P., \& Büttgenbach, T. H. 1995, A\&A, 297, 5

van Dishoeck, E. F., \& Blake, G. A. 1998, ARA\&A, 36, 317

Walmsley, C. M., Natta, A., Oliva, E., \& Testi, L. 2000, A\&A, 364, 301

Wilson, T. L., Mauersberger, R., Gensheimer, P. D., Muders, D., \& Bieging, J. H. 1999, ApJ, 525, 343

Young Owl, R. C., Meixner, M., Wolfire, M., Tielens, A. G. G. M., \& Tauber, J. 2000, ApJ, 540, 886 